\section{Nanosurf AG}

Nanosurf was founded in 1997 in Liestal, Switzerland, and has since become one of the most established and trusted AFM brands in the market today. True to its mission of being a customer centric company, Nanosurf has direct sales, service, and support offices in China, Germany, India, Singapore, the UK and the US.

From our modest beginnings of developing an STM that can achieve atomic resolution on a coffee table, to the current day, where we offer a range of AFMs and associated technologies, Nanosurf has always depended on innovation as the corner stone for growth. Some of the innovative technologies that we have introduced to the market include the FluidFM ${ }^{\circledR}$ (in partnership with Cytosurge) and Cytomass Monitor (in partnership with ETH Zurich).

Our current product line includes:

- DriveAFM: Nanosurf's new flagship instrument utilizes the latest technology to deliver stable, high-end performance. It was designed to fulfill the needs of top-notch research, today and in the future. The DriveAFM includes many unique features such as photothermal actuation (CleanDrive) for stable excitation of the cantilever in air or liquid, a direct drive scanner and a fully motorized system that can be operated on an inverted optical microscope or as a stand-alone system.

- FlexAFM: The FlexAFM combines versatility and performance for researchers both in life sciences and material science. By advancing key technologies and intelligent design, The FlexAFM has become on one of the most versatile and flexible AFMs allowing for a large variety of applications to be handled with ease. The FlexAFM can also be easily integrated into any inverted optical microscope with ease, thereby enabling a variety of life science applications.

- CoreAFM: The CoreAFM is the result of intelligently combining the core components of AFM to achieve a user-friendly research-grade AFM that provides the best value to researchers. The compact design of the CoreAFM includes all the essential functions of modern AFM; all you need to do connect the controller and plug-in power to start using the CoreAFM.

- Alphacen 300: The Alphacen 300 is Nanosurf's large sample AFM. It was designed to handle samples as large as $300 \mathrm{~mm}$ and as heavy as $45 \mathrm{Kg}$. The Alphacen 300 includes a sample stage that can move $300 \mathrm{~mm}$ x $300 \mathrm{~mm}$ in XY and a Z-stage that travels $50 \mathrm{~mm}$. The Alphacen 300 includes powerful automation software that allows the user to preselect locations of interest, either on an optical image or a stage map, and let the system collect the images with no user intervention.

- Customization: Nanosurf is the market leader for custom developed systems for large, heavy or non-planar samples. Over the past years our team has built a substantial knowledge base developing these custom stages for various customers. Nanosurf offers the unique service of designing and developing custom AFM systems and stages that will allow you to perform your measurements with minimal disruption of your established process.

\section{r nanosurf}
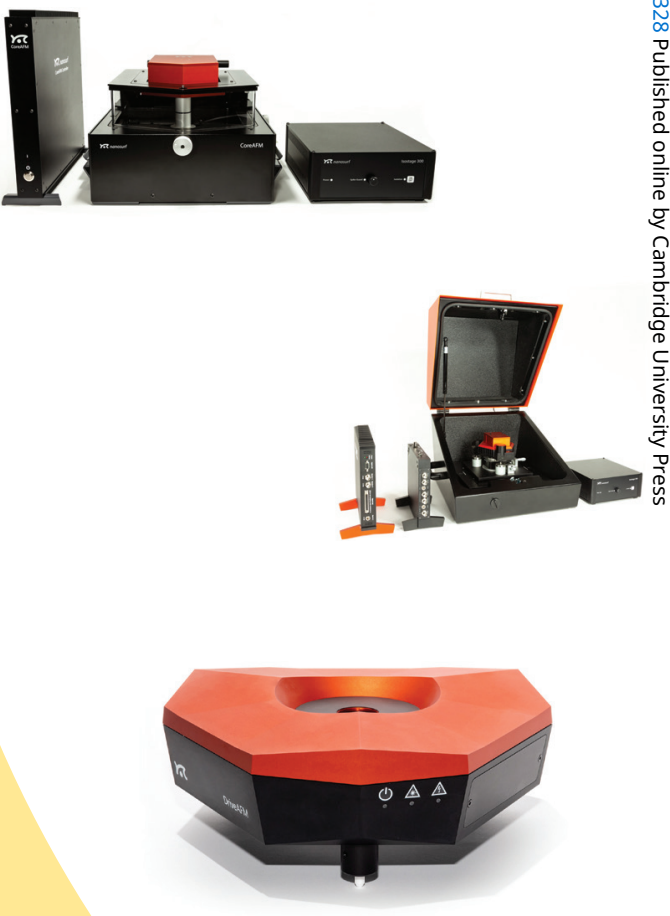

\section{How to find us}

\author{
Nanosurf AG, Gräubernstrasse 12, \\ CH-4410 Liestal, Switzerland \\ Tel: +41619274747 \\ Fax: +41 619274700 \\ Email: info@nanosurf.com \\ www.nanosurf.com \\ Locations: Liestal, Switzerland | Langen, \\ Germany | Bracknell, UK | Woburn, MA, \\ USA | Shanghai, China | Hyderabad, India \\ | Singapore
}

